\title{
Multiplicative relations of points on algebraic groups
} by

\author{
Yuval Z. FLICKER and Piotr KRASOŃ \\ Presented by Jerzy KACZOROWSKI
}

\begin{abstract}
Summary. Our aim here is to restructure the area of multiplicative relations on points and congruences, by proposing a novel conjecture in the context of general reductive linear algebraic groups. To support our conjecture we check it in a few elementary but new cases, and claim this extends classical work in number theory on multiplicative relations on points and congruences, initiated by Skolem and Schinzel, which we rephrase group-theoretically as Hasse principles on commutative linear algebraic groups, or tori, so that a part of it becomes the abelian case of our conjecture. Our conjecture can then be viewed as an extension to general - not necessarily commutative - reductive linear algebraic groups of a part of Schinzel's result. We relate it to the Erdös support problem. To motivate our conjecture from another perspective we note that analogues have been extensively developed for abelian varieties. We give a short account of this, and state a question on the "detecting linear dependence" problem.
\end{abstract}

1. Introduction. The aim of this article is to restructure the area of multiplicative relations on points and congruences, by proposing a novel conjecture in the context of general reductive linear algebraic groups. To motivate and support the conjecture we present several results of multiplicative local-to-global nature, originating on one hand from work of Skolem in 1937 generalized by Schinzel in 1975, and on the other from a question of Erdős in 1988, answered by Corrales-Rodrigáñez and Schoof in 1997. We phrase the Schinzel-Skolem question group-theoretically as a Hasse-principle type result on a split torus. The conditions - that we express precisely and explicitly - for the existence of a global solution involve

2010 Mathematics Subject Classification: 11-02, 14G05, 11D61, 11D57, 11J61, 14G25, $11 \mathrm{G} 35$.

Key words and phrases: linear algebraic groups, rational points, multiplicative relations, Hasse principle.

Received 14 March 2017; revised 18 July 2017.

Published online 12 October 2017. 
almost all ideals in the ring of integers of the number field in question; making the local assumptions only at the prime ideals does not suffice. We ask whether the result extends to reductive (connected) linear algebraic groups which are not commutative. We then propose a unifying conjecture in the context of general reductive linear algebraic groups. This conjecture is the key contribution of this paper. To support our conjecture we prove it in a few elementary - but new - noncommutative cases. Further we claim this extends the aforementioned classical work in number theory on multiplicative relations on points and congruences, which we rephrase grouptheoretically as Hasse principles on commutative linear algebraic groups, or tori, so this classical work - or rather its integral case-becomes the abelian case of our conjecture. Our conjecture can then be viewed as an extension to general - not necessarily commutative - reductive linear algebraic groups, or rather their groups of integral points. This is all in Section 2.

The Erdős problem - also a Hasse-principle type question for a commutative group - is recalled in Section 3. It was extended in 2004 by Khare and D. Prasad to the context of $\mathrm{SL}(2, \mathbb{Z})$, and in Section 5 here to $\operatorname{SL}(n, \mathcal{O})$ and inner automorphisms, where $\mathcal{O}$ is the ring of integers of a number field $F$. An extension to $G(\mathcal{O})$ for a reductive (connected) $\mathcal{O}$-group is begging to be discovered.

The results concerning commutative linear algebraic groups (multiplicative tori) have been developed extensively in the analogous context of abelian varieties, in many papers, notably in works of the second named author and his collaborators Banaszak and Gajda, by Larsen, Weston, Jossen, Perucca, Khare and Prasad. Section 4 gives a short account of this. More precisely, we describe there the "support" problem and the "detecting linear dependence" problem for abelian varieties. We also discuss a sufficient condition of [BK11] for the latter problem to have a positive solution, and formulate a general problem on possible removal of the torsion ambiguity in that solution.

Our main conjecture 2.2 and the work of Section 2-opens a new direction, in the context of reductive groups, extending classical numbertheoretical studies that we interpret as the commutative case, an analogue of the case of abelian varieties.

To motivate the conjecture of Section 2 we gather in Sections 3-5 a few relevant results known on linear algebraic groups, and draw attention to the question of extension to general noncommutative reductive groups, in the hope of stimulating research in this new direction.

2. A result of Schinzel. To state Schinzel's result precisely we write its conditions explicitly, in particular specify the $D>0$ that appears in the assumption. 
Let $F$ be a number field. Denote by $\mathcal{O}$ its ring of integers. Let $a_{i}$ be finitely many elements in $F^{\times}$. Decompose the fractional ideal $\left(a_{i}\right)$ as a reduced product of primes $\prod_{s} \mathfrak{p}_{i s} / \prod_{t} \mathfrak{q}_{i t}$, and denote by $p_{i s}, q_{i t}$ the characteristics of the residue fields $\mathcal{O} / \mathfrak{p}_{i s}$ and $\mathcal{O} / \mathfrak{q}_{i t}$. Write $D=D\left(\left\{a_{i}\right\}\right)$ for the product $\prod_{i}\left(\prod_{s} p_{i s} \cdot \prod_{t} q_{i t}\right)$, and define the ring $\mathcal{O}_{(D)}$ to be $\mathcal{O}\left[1 / p_{i s}, 1 / q_{i t} ; i, s, t\right]$, generated over $\mathcal{O}$ by the inverses of all $p_{i s}$ and $q_{i t}$ in $D$. Then for each ideal $\mathfrak{m}$ in $\mathcal{O}$ that is prime to $D$, we have $a_{i} \in \mathcal{O}_{(D)}$ and $a_{i}(\bmod \mathfrak{m})$ in $\mathcal{O}_{(D)} / \mathfrak{m} \mathcal{O}_{(D)}$. We write $a \equiv b(\bmod \mathfrak{m})$ if $a, b$ lie in $\mathcal{O}_{(D)}$ and $a-b \in \mathfrak{m} \mathcal{O}_{(D)}$.

Theorem 3 of Schinzel [Sch75] asserts:

THEOREM 2.1. Let $F$ be a number field. Let $T$ be the torus $\mathbb{G}_{\mathrm{m}}^{n}$. Fix $t_{1}, \ldots, t_{r}, t_{0}$ in $T(F)$. Let $D=D\left(\left\{t_{i}: 0 \leq i \leq r\right\}\right)>0$ be the rational integer associated as above with the components of the $t_{i}$. Suppose that for almost every ideal $\mathfrak{m}$ in the ring $\mathcal{O}$ of integers of $F$ that is prime to $D$, there are $x_{1, \mathfrak{m}}, \ldots, x_{r, \mathfrak{m}}$ in $\mathbb{Z}$ such that $t_{1}^{x_{1, \mathfrak{m}}} \cdots t_{r}^{x_{r, \mathfrak{m}}} \equiv t_{0}(\bmod \mathfrak{m})$. Then there are $x_{1}, \ldots, x_{r} \in \mathbb{Z}$ with $t_{1}^{x_{1}} \cdots t_{r}^{x_{r}}=t_{0}$.

In fact Schinzel writes $t_{j}=\left(a_{1 j}, \ldots, a_{n j}\right)(1 \leq j \leq r)$ and $t_{0}=\left(a_{10}\right.$, $\left.\ldots, a_{n 0}\right)$, assumes the existence of $x_{j, \mathfrak{m}}(1 \leq j \leq r)$ with $a_{i 1}^{x_{1, \mathfrak{m}}} \cdots a_{i r}^{x_{r, \mathfrak{m}}} \equiv$ $a_{i 0}(\bmod \mathfrak{m})$ for all $i(1 \leq i \leq n)$, and for all $\mathfrak{m}$ prime to $D$ where his $D>0$ is any positive rational integral multiple of the $D>0$ we described, he deduces the existence of $x_{j}(1 \leq i \leq r)$ with $a_{i 1}^{x_{1}} \cdots a_{i r}^{x_{r}}=a_{i 0}$. He notes that when $n=1$, it suffices to make the assumption only for prime ideals $\mathfrak{m}=\mathfrak{p}$ Sch75, Theorem 2], and a weaker form of this is due to Skolem [Sk37]. A shorter proof of the Skolem case is due to Khare [Kh03, Proposition 3]. Further Schinzel provides an example (with $n=2, r=3$, [Sch75, pp. 419-420]) to show that it does not suffice to make the assumption for "all primes $\mathfrak{p}$ " instead of "all ideals $\mathfrak{m}$ prime to $D$ ". For convenience of the reader and for clarity of the discussion we reproduce this example in Section 4.

There has been much progress in developing an analogue of this result where abelian varieties replace the abelian linear algebraic group $T$ - see our discussion in Section 4. Does the theorem extend to noncommutative (linear) algebraic groups, especially reductive connected groups?

Let us try to test the natural conjecture, extending the integral part of Schinzel's Theorem 2.1 to other linear groups. It may take various forms, one of which would be the following.

ConjeCture 2.2. Let $F$ be a number field. Let $G$ be a linear algebraic group over $\mathcal{O}$, viewed as a subgroup of some matrix group $\operatorname{GL}(n)$. Fix $g_{0}, g_{1}, \ldots, g_{r}$ in $G(\mathcal{O})$. Let $D>0$ be the rational integer which is associated with the coefficients of $g_{0}, g_{1}, \ldots, g_{r}$. Suppose for almost every ideal $\mathfrak{m}$ in the ring $\mathcal{O}$ of integers of $F$ that is prime to $D$, there are $x_{1, \mathfrak{m}}, \ldots, x_{r, \mathfrak{m}} \in \mathbb{Z}$ such that $g_{1}^{x_{1, \mathfrak{m}}} \cdots g_{r}^{x_{r, \mathfrak{m}}} \equiv g_{0}(\bmod \mathfrak{m})$. Then there are $x_{1}, \ldots, x_{r} \in \mathbb{Z}$ with $g_{1}^{x_{1}} \cdots g_{r}^{x_{r}}=g_{0}$. 
Perhaps our $D>0$ can be replaced by any positive rational integral multiple of itself.

For $g, g^{\prime}$ in $G(\mathcal{O}), G \subset \mathrm{GL}(n)$, by $g^{\prime} \equiv g(\bmod \mathfrak{m})$ we mean that $g^{\prime} g^{-1} \in$ $G(\mathcal{O})$ lies in $I+\mathfrak{m} M(n, \mathcal{O})$, where $M(n)$ denotes the ring of $n \times n$ matrices. The assumption that $g_{0}, g_{1}, \ldots, g_{r}$ are integral, thus they are in $G(\mathcal{O})$, is motivated by the integrality assumption in the analogous case of abelian varieties (see Section 4), where the general case is reduced to the integral case by using the Néron model. In the case of linear algebraic groups there is no such reduction. In fact most of Theorem 2.1 is an assertion on nonunit $t_{i}$, in a direction perpendicular to our integral case. For example, when $F$ is $\mathbb{Q}$ the only units are \pm 1 .

A crucial case of the conjecture is that of $G=\mathrm{SL}(2)$. A simpler case is the Borel (upper triangular) subgroup $B=A U$. Even further, we may take $G$ to be the unipotent radical $U$ of $B$. If $g_{i}=\left(\begin{array}{cc}1 & u_{i} \\ 0 & 1\end{array}\right)$, when $F=\mathbb{Q}$ a variant of Conjecture 2.2 states the following.

Proposition 2.3. Suppose $u_{0}, u_{1}, \ldots, u_{r}$ are nonzero rational integers. Let $D>0$ be an integer prime to $u=\operatorname{gcd}\left(u_{1}, \ldots, u_{r}\right)$. Suppose for each $m>1$ prime to $D$ there are integers $x_{i, m}(1 \leq i \leq r)$ with $x_{1, m} u_{1}+$ $\cdots+x_{r, m} u_{r} \equiv u_{0}(\bmod m)$. Then there are integers $x_{i}(1 \leq i \leq r)$ with $x_{1} u_{1}+\cdots+x_{r} u_{r}=u_{0}$.

Proof. Since $u=\operatorname{gcd}\left(u_{1}, \ldots, u_{r}\right)$, there are $x_{i}^{\prime} \in \mathbb{Z}$ with $\sum_{1 \leq i \leq r} x_{i}^{\prime} u_{i}=u$. If $u \mid u_{0}$ then $\sum_{1 \leq i \leq r}\left(\frac{u_{0}}{u} x_{i}^{\prime}\right) u_{i}=u_{0}$.

If $u$ does not divide $u_{0}$, replace $u_{i}$ by $u_{i} /\left(u, u_{0}\right)(0 \leq i \leq r)$ to get $\left(u, u_{0}\right)=1$. Take $m \mid u$. Then $\left(m, u_{0}\right)=1$ and $(m, D)=1($ from $(u, D)=1)$. Then the left side of the congruence $\sum_{1 \leq i \leq r} x_{i m} u_{i} \equiv u_{0}(\bmod m)$ is zero, leading to a contradiction as $u_{0}$ is not zero $\bmod m$.

Here is an alternative formulation. Let $S_{m}$ be the statement

$$
x_{1 m} u_{1}+\cdots+x_{r m} \equiv u_{0}(\bmod m) \text { has a solution with } x_{i m} \in \mathbb{Z}, 1 \leq i \leq r .
$$

Let $S$ be the statement

$$
x_{1} u_{1}+\cdots+x_{r} u_{r}=u_{0} \text { has a solution with } x_{i} \in \mathbb{Z}, 1 \leq i \leq r .
$$

Denote by $(\ldots)$ the ideal in $\mathbb{Z}$ generated by .... Then

$$
S_{m} \Leftrightarrow\left\{u_{0} \in\left(u_{1}, \ldots, u_{r}, m\right)\right\}, \quad S \Leftrightarrow u_{0} \in\left(u_{1}, \ldots, u_{r}\right) .
$$

Our claim is

$$
\bigcap_{m}\left(u_{1}, \ldots, u_{r}, m\right)=\left(u_{1}, \ldots, u_{r}\right),
$$

where $m$ ranges over $\mathbb{Z}_{>0}$ with $(m, D)=1$. As $\mathbb{Z}$ is a $\mathrm{PID},\left(u_{1}, \ldots, u_{r}\right)=(u)$ for some $u \in \mathbb{Z}_{>0}$. Then $\left(u_{1}, \ldots, u_{r}, m\right)=\left(u_{1}, \ldots, u_{r}\right)+(m)=(u)+(m)$. 
Our claim is then

$$
\bigcap_{m}((u)+(m))=(u)
$$

If $u>1$ and $D=u$, then $(u)+(m)=(1)=\mathbb{Z}$ for every $m$ prime to $D$, so $\bigcap_{m}((u)+(m))=(1) \neq(u)$. But if $D$ is prime to $u$, as we assume, we can take $m=u$ and the claim holds.

The second variant of the proof of this result holds for a number field $F$ of class number one, where the $u_{i}$ are integral in $F$. It will be interesting to prove Proposition 2.3 for any number field $F$, of any class number.

When $G$ is a Heisenberg group, say the unipotent radical of the upper triangular subgroup of $\mathrm{SL}(3)$, the following question comes up, first again in the context of integers. Suppose that, instead of $u_{0}, u_{1}, \ldots, u_{r}$, we have two sequences of integers $u_{0}, u_{1}, \ldots, u_{r}$ and $v_{0}, v_{1}, \ldots, v_{r}$; and for $D$ prime to $\left(u_{1}, \ldots, u_{r}\right)$ and $\left(v_{1}, \ldots, v_{r}\right)$, for each $m$ prime to $D$ the equations $x_{1, m} u_{1}+$ $\cdots+x_{r, m} u_{r} \equiv u_{0}(\bmod m)$ and $x_{1, m} v_{1}+\cdots+x_{r, m} v_{r} \equiv v_{0}(\bmod m)$ are solvable, with the same integral $x$ 's. Thus $x_{i, m}$ are the same for the $u$ 's and for the $v$ 's. Then there is a solution to $x_{1} u_{1}+\cdots+x_{r} u_{r}=u_{0}$ and to $y_{1} v_{1}+\cdots+y_{r} v_{r}=v_{0}$. We should be able to choose $y_{i}=x_{i}$. Is this true? Yes it is (at least when $D=1$ ), even more generally, in the context of $G$ being the unipotent radical $U$ of the upper triangular subgroup of $\operatorname{SL}(n)$. Regarding the above diagonal, that is, the derived group $U /[U, U]$ of $U$, we have, in the context of $F=\mathbb{Q}$ :

Proposition 2.4. Suppose $u_{0, j}, u_{1, j}, \ldots, u_{r, j}(1 \leq j<n)$ are nonzero integers. Fix $m_{0} \geq 1$. Suppose for each $m \geq m_{0}$ there are integers $x_{i, m}$ $(1 \leq i \leq r)$ with $x_{1, m} u_{1, j}+\cdots+x_{r, m} u_{r, j} \equiv u_{0, j}(\bmod m)$ for all $j$. Then there are integers $x_{i}(1 \leq i \leq r)$ with $x_{1} u_{1, j}+\cdots+x_{r} u_{r, j}=u_{0, j}$ for all $j$, $1 \leq j<n$.

Proof. The theorem of Skolem [Sk37, Hilfsatz 2, p. 8] asserts that a system of linear equations $A X=B$, where $A$ is an integral matrix and $B$ an integral vector, has an integral solution if and only if it is solvable mod $m$ for all moduli $m>1$. By our assumptions the system of linear equations

$$
\begin{aligned}
& x_{1, j, m} u_{1, j}+x_{2, j, m} u_{2, j}+\cdots+x_{r, j, m} u_{r, j}=u_{0, j} \quad(1 \leq j<n), \\
& x_{i, j, m}=x_{i, 1, m} \quad(1 \leq i \leq r, 2 \leq j<n)
\end{aligned}
$$

has a solution for any $m$. Therefore by the Skolem theorem, the system

$$
\begin{aligned}
& x_{1, j,} u_{1, j}+x_{2, j} u_{2, j}+\ldots x_{r, j} u_{r, j}=u_{0, j} \quad(1 \leq j<n), \\
& x_{i, j}=x_{i, 1} \quad(1 \leq i \leq r, 2 \leq j<n)
\end{aligned}
$$

has an integral solution. We set $x_{i}=x_{i, 1}$ and the proposition follows.

For $m \geq m_{0}$, note that if $1<m<m_{0}$, then $m m_{0}>m_{0}$, we have a congruence $\bmod m m_{0}$ by assumption, and this can be reduced $\bmod m$. 
3. The support problem. A question that Paul Erdős asked for $F=\mathbb{Q}$ was answered by Corrales-Rodrigáñez and Schoof [CRS97, Theorem 1].

TheOREm 3.1. Let $F$ be a number field. Fix $x, y$ in $F^{\times}$. Suppose that for almost all prime ideals $\mathfrak{p}$ in the ring $\mathcal{O}$ of integers of $F$, and for all positive rational integers $n$, we have $y^{n} \equiv 1(\bmod \mathfrak{p})$ if $x^{n} \equiv 1(\bmod \mathfrak{p})$. Then $y$ is a power of $x$.

This result implies that if the congruence $y^{n} \equiv 1(\bmod \mathfrak{p})$ is equivalent to $x^{n} \equiv 1(\bmod \mathfrak{p})$, then $x=y^{ \pm 1}$ or both $x$ and $y$ are roots of unity. When $F=\mathbb{Q}$ and $x, y$ are positive integers, this answers positively the question of Erdős from 1988. Theorem 3.1 is false for the additive group $\mathbb{G}_{a}$, and hence for the groups $\operatorname{GL}(n)$ with $n \geq 2$, or any reductive nonanisotropic linear algebraic group of rank $\geq 2$.

We review the proof of the theorem. Denote by $\zeta_{n}$ a primitive $n$th root of 1 and by $\mu_{n}$ the group generated by $\zeta_{n}$ in $\mathbb{C}$. Set $\zeta_{4}=i$. To prove the theorem we need

LEMmA 3.2. Let $q$ be a power of a rational prime $\ell$. If $\ell=2$, assume that $i \in F$. Denote by $\sigma$ a generator of the cyclic group $G_{q}=\operatorname{Gal}\left(F\left(\zeta_{q}\right) / F\right)$. Let $N_{q}: F\left(\zeta_{q}\right)^{\times} \rightarrow F^{\times}$be the norm map. Then:

(i) For $\zeta \in \mu_{q}$ we have: $N_{q}(\zeta)=1$ if and only if $\zeta=\sigma(\xi) / \xi$ for some $\xi \in \mu_{q}$.

(ii) The natural map $F^{\times} / F^{\times q} \rightarrow F\left(\zeta_{q}\right)^{\times} / F\left(\zeta_{q}\right)^{\times q}$ is injective.

Proof. (i) The group $G_{q}$ is isomorphic to a subgroup $H$ of $(\mathbb{Z} / q \mathbb{Z})^{\times}=$ $\operatorname{Aut}\left(\mu_{q}\right)$. If $\ell=2, H$ is contained in $\left\{x \in(\mathbb{Z} / q \mathbb{Z})^{\times}: x \equiv 1(\bmod 4)\right\}$. Hence $H$ is cyclic. Denote its order by $d$. Let $a \in(\mathbb{Z} / q \mathbb{Z})^{\times}$be a generator of $H$. Set

$$
B=\left\{x \in \mathbb{Z} / q:\left(1+a+a^{2}+\cdots+a^{d-1}\right) x=0\right\}, \quad Z=(1-a)(\mathbb{Z} / q \mathbb{Z}) .
$$

Then $Z \subset B$. The homomorphism $\psi(x)=\zeta_{q}^{x}, \psi: B \rightarrow\left\{\zeta \in \mu_{q}: N_{q}(\zeta)=1\right\}$, defines an isomorphism

$$
\psi: B / Z \stackrel{\sim}{\rightarrow}\left\{\zeta \in \mu_{q}: N_{q}(\zeta)=1\right\} /\left\{\zeta / \sigma(\zeta): \sigma \in G_{q}\right\},
$$

where $\sigma \zeta=\zeta^{a}$. Indeed, $\psi$ is onto because if $1=N_{q}(\zeta)=\zeta \cdot \zeta^{a} \cdots \zeta^{a^{d-1}}$ and $\zeta=\zeta_{q}^{x}$, then $x \in B$. Since $\psi(Z) \subset\left\{\zeta / \sigma(\zeta): \sigma \in G_{q}\right\}$, the map $\psi$ factors through $B / Z$. The kernel of the factored $\psi$ is trivial since if $\psi(x)=$ $\zeta / \sigma(\zeta)=\zeta_{q}^{y(1-a)}$ for $\zeta=\zeta_{q}^{y}$, as $\psi(x)=\zeta_{q}^{x}$ we have $x=y(1-a) \in Z$.

But $Z=B$. Indeed, if $x \in B$ then $x+a x+a^{2} x+\cdots+a^{d-1} x \equiv 0(\bmod q)$, hence $a x+a^{2} x+\cdots+a^{d} x \equiv 0(\bmod q)$, and $x \equiv x+a\left(x+a x+a^{2} x+\cdots+\right.$ $\left.a^{d-1} x\right)(\bmod q)$. Set $u=-\left(a x+a^{2} x+\cdots+a^{d-1} x\right)$. Then $x \equiv u(\bmod q)$, so $x \equiv x-a u \equiv(1-a) u$ lies in $Z$. Thus (i) follows. 
(ii) If $t \in F^{\times}$equals $s^{q}$ for some $s \in F\left(\zeta_{q}\right)$, then $s^{q}=\sigma(s)^{q}$, so $\sigma(s) / s$ is a $q$ th root of 1 which is in the kernel of the norm $N_{q}$. By (i), there is some $\xi \in \mu_{q}$ with $\sigma(s) / s=\xi / \sigma(\xi)$. Hence $s \xi \in F$. But $t=s^{q}=(s \xi)^{q}$, so (ii) follows.

REMARK 3.3. Note that (i) gives a direct proof of Hilbert's Theorem 90 for the extension $F\left(\zeta_{q}\right) / F$, that is, $H^{1}\left(G_{q}, \mu_{q}\right)=H^{1}\left(G_{q}, F\left(\zeta_{q}\right)^{\times}\right)=1$, and thus $\psi$ is an isomorphism [Rot09, Theorem 9.32 and Corollary 9.33]. One can give a more cohomological proof of (i) using this more general theorem. Similarly (ii) is a consequence of Hilbert's Theorem 90 [Se97, II, $\S 1.2$, Proposition 1 and Corollary, p. 72] and the restriction-inflation sequence Se97, I, $\S 2.6\left(\right.$ b) , p. 15] applied to the normal subgroup $\operatorname{Gal}\left(\bar{F} / F\left(\zeta_{q}\right)\right)$ of $\left.\operatorname{Gal}(\bar{F} / F)\right)$.

Proof of Theorem 3.1. Replacing $F$ by $F(i)$ we may assume that $i \in F$. Let $T$ be the set of bad prime ideals $\mathfrak{p}$ of $F$ : the Archimedean primes, those with $|x|_{\mathfrak{p}} \neq 1$ or $|y|_{\mathfrak{p}} \neq 1$, and those for which the assumption of the theorem does not hold. Then $T$ is a finite set.

We shall use [Bi67, Lemma 2.5, p. 91]. Let $n$ be a positive rational integer.

Lemma 3.4. The discriminant of $F(\sqrt[n]{a})$ over $F$ divides $n^{n} a^{n-1}$. The ideal $\mathfrak{p}$ of $\mathcal{O}$ is unramified if $\mathfrak{p}$ does not divide na. Let $f \geq 1$ be the least integer such that there is $x$ in $\mathcal{O}$ with $a^{f} \equiv x^{n}(\bmod \mathfrak{p})$. Then $f$ is the degree of the residue field.

So in particular we have

Corollary 3.5. The ideal $\mathfrak{p}$ in $\mathcal{O}$ splits completely in $F\left({ }^{n} \sqrt{a}\right) / F$ precisely when $f=1$, namely $a$ is an nth power in the residue field $k_{\mathfrak{p}}=\mathcal{O} / \mathfrak{p}$.

Recall also the well-known [Ne99, Corollary 10.4, p. 63]

Lemma 3.6. An odd rational prime $p$ splits completely in $\mathbb{Q}\left(\zeta_{n}\right)$ if and only if $p \equiv 1(\bmod n)$.

We use these with $n=q$ a power of a rational prime $\ell$. Denote by $\bar{F}$ a fixed separable algebraic closure of $F$. Consider the number fields $F\left(\zeta_{q}, \sqrt[q]{x}\right)$ and $F\left(\zeta_{q}, \sqrt[q]{y}\right)$ in $\bar{F}$. Let $\mathfrak{p} \notin T$ be a prime ideal in $\mathcal{O}$. Let $p=\left|\mathbb{F}_{\mathfrak{p}}\right|$ denote the cardinality of the residue field $\mathbb{F}_{\mathfrak{p}}=\mathcal{O} / \mathfrak{p}$. Suppose $\mathfrak{p}$ splits completely in $F\left(\zeta_{q}, \sqrt[q]{x}\right)$. Thus $p \equiv 1(\bmod q)$ by Lemma 3.6 , and $x \in \mathbb{F}_{\mathfrak{p}}^{q}$ by Corollary 3.5 . Hence $x^{(p-1) / q} \equiv 1(\bmod \mathfrak{p})$, and so $y^{(p-1) / q} \equiv 1(\bmod \mathfrak{p})$ by the assumption of the theorem. Now $\mathbb{F}_{\mathfrak{p}}^{\times}$is cyclic of order $p-1$, hence $y$ is a $q$ th power modulo $\mathfrak{p}$. Consequently, $\mathfrak{p}$ splits completely in $F\left(\zeta_{q}, \sqrt[q]{y}\right)$.

By the Frobenius Density Theorem, we have $(*): F\left(\zeta_{q}, \sqrt[q]{y}\right) \subset F\left(\zeta_{q}, \sqrt[q]{x}\right)$. Consider the map

$$
\theta: F\left(\zeta_{q}\right)^{\times} / F\left(\zeta_{q}\right)^{\times q} \rightarrow \operatorname{Hom}\left(\operatorname{Gal}\left(\bar{F} / F\left(\zeta_{q}\right)\right), \mu_{q}\right),
$$


$\theta(t)=\varphi_{t}$, where $\varphi_{t}(\sigma)=\sigma(\sqrt[q]{t}) / \sqrt[q]{t}$ for $\sigma \in \operatorname{Gal}\left(\bar{F} / F\left(\zeta_{q}\right)\right)$. From $(*)$ we have $\operatorname{ker} \varphi_{x} \subset \operatorname{ker} \varphi_{y}$.

Consider the commutative diagram

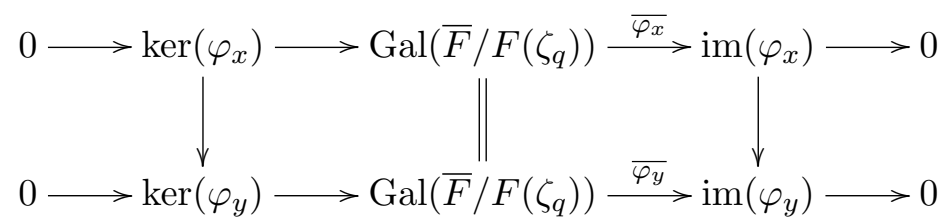

As the group $\operatorname{im}\left(\varphi_{x}\right) \subset \mu_{q}$ is cyclic, the map $\psi$ is just exponentiation by an integer $d$. Hence $\varphi_{y}=\varphi_{x}^{d}$. The Kummer map $\theta$ is injective, hence $y=x^{d}$ in the group $F\left(\zeta_{q}\right)^{\times} / F\left(\zeta_{q}\right)^{\times q}$. By Lemma 3.1(ii), we have $y=x^{d}$ in $F^{\times} / F^{\times q}$.

Let $\mathcal{O}_{T}^{\times}$be the multiplicative group of $T$-units in $F^{\times}$. Set $A=\mathcal{O}_{T}^{\times} / x^{\mathbb{Z}}$. We have seen that $y \in A$ lies in fact in $A^{q}$ for every prime power $q$. By the Dirichlet Unit Theorem the group $A$ is finitely generated. Hence $\bigcap_{q} A^{q}$ is $\{1\}$. So the image of $y$ in $A$ is trivial, thus $y=x^{a}$ for some $a \in \mathbb{Z}$. The theorem follows.

We next explain Proposition 1 of [KP04] and elaborate on its proof.

COROLlaRY 3.7. Let $\phi: \mathcal{O}^{\times} \rightarrow \mathcal{O}^{\times}$be a homomorphism which reduces mod $\mathfrak{p}$ for almost all prime ideals $\mathfrak{p}$ in the ring $\mathcal{O}$ of integers of the number field $F$, and thus defines $\phi_{\mathfrak{p}}:(\mathcal{O} / \mathfrak{p})^{\times} \rightarrow(\mathcal{O} / \mathfrak{p})^{\times}$for almost all $\mathfrak{p}$. Then there is an integer $m \in \mathbb{Z}$ with $\phi(x)=x^{m}$.

Proof. That $\phi$ reduces for almost all $\mathfrak{p}$ means that for almost all $\mathfrak{p}$, if $x^{n} \equiv 1(\bmod p)$ then $\phi(x)^{n} \equiv 1(\bmod p)$. By Theorem 3.1 we conclude that $\phi(x)=x^{d_{x}}$. We have to show that $d_{x} \in \mathbb{Z}$ does not depend on $x$. For this we use Dirichlet's Unit Theorem. We choose a basis of fundamental units. If there is a nontorsion part in $\mathcal{O}^{\times}$then we immediately see that $d_{x}$ has to be independent of $x$. If $\mathcal{O}^{\times}$is torsion, then it has to be finite cyclic, and any homomorphism is thus given by some power.

4. Case of abelian varieties. Does the support problem extend to the context of abelian varieties? This question was considered by the authors of [CRS97, who gave a positive answer for elliptic curves. The problem was solved in [BGK03] for some classes of abelian varieties for which the images of the $l$-adic representations are well understood. The exact statement is as follows.

TheOREM 4.1. Let $A$ be an abelian variety of dimension $g \geq 1$, defined over a number field $F$, and such that $A$ satisfies one of the following conditions:

(1) A has a nondegenerate CM type with $\operatorname{End}_{F} \otimes \mathbb{Q}$ equal to a $\mathrm{CM}$ field $E$ such that the Hilbert class field $E^{H}$ of $E$ is contained in $F$. 
(2) A is simple, principally polarized, with real multiplication by a totally real field $E=\operatorname{End}_{F}(A) \otimes \mathbb{Q}$ such that $E^{H} \subset F$ and the field $F$ is sufficiently large. Assume also that $\operatorname{dim} A=$ eh, where $e=[E: \mathbb{Q}]$ and $h$ is odd, or $A$ is simple, principally polarized, such that $\operatorname{End}_{F}(A)=\mathbb{Z}$ and $\operatorname{dim} A$ is 2 or 6 .

Let $P, Q$ be two nontorsion elements of the group $A(F)$. Assume that for almost every prime $v$ of $\mathcal{O}_{F}$ and for every positive integer $m$ the following condition holds in $A_{v}\left(k_{v}\right)$ :

$$
m r_{v}(P)=0 \quad \text { implies } m r_{v}(Q)=0 .
$$

Then there exist $a \in \mathbb{Z}-\{0\}$ and $f \in \mathcal{O}_{E}-\{0\}$ such that $a P+f Q=0$ in $A(F)$.

A solution to the support problem for any absolutely simple abelian variety defined over a number field $F$ was given in KP04. M. Larsen [La03] gave a general solution.

The support problem is related to a problem posed by W. Gajda: does the analogue of Schinzel's theorem-for one exponential congruence and only prime ideals Sch75, Theorem 2] —hold in the case of abelian varieties? The precise question is the following.

QUESTION 4.2. Let $A$ be an abelian variety over a number field $F$. Let $v$ be a non-Archimedean place of $F$. Denote the residue field by $k_{v}$. Let $\operatorname{red}_{v}: A(F) \rightarrow A\left(k_{v}\right)$ be the reduction map at $v$. Let $\Lambda$ be a subgroup of $A(F)$. Suppose that $x$ is a point of $A(F)$ such that $\operatorname{red}_{v} x$ lies in $\operatorname{red}_{v} \Lambda$ for almost all places $v$ of $F$. Does it follow that $x$ lies in $\Lambda$ ?

When $\Lambda$ is cyclic and $A$ is an abelian group scheme, this question was independently posed by E. Kowalski [Ko03]. The question is called the "detecting linear dependence problem". T. Weston [We03] obtained a first result in this direction:

THEOREM 4.3. Let $A$ be an abelian variety over a number field $F$. Assume that $\operatorname{End}_{F} A$ is commutative. Let $\Lambda$ be a subgroup of $A(F)$. Suppose that $x \in A(F)$ is such that $\operatorname{red}_{v} x \in \operatorname{red}_{v} \Lambda$ for almost all places $v$ of $F$. Then $x \in \Lambda+A(F)_{\text {tor }}$.

The reduction map $r_{v}: A(F) \rightarrow A_{v}\left(k_{v}\right)$ is constructed for a prime $v$ of good reduction in terms of the Néron model $\mathcal{A}$ of $A$ (see [BLR90]) using the property that $\mathcal{A}\left(\mathcal{O}_{F}\right)=A(F)$.

Theorem 4.3 has two limitations. First, the ring of endomorphisms is assumed to be commutative. This excludes abelian varieties that have simple factors of multiplicity greater than 1 . Second, the result is up to torsion.

An affirmative answer to Question 4.1 - without torsion ambiguity — was given in [BGK05], for principally polarized abelian varieties with $\operatorname{End}_{\bar{F}}(A)$ 
$=\mathbb{Z}$ and such that $g=\operatorname{dim} A$ is odd or $g=2$ or $g=6$. It was also shown there that for any abelian variety and any free $\operatorname{End}_{F}(A)$-module $\Lambda$, and any point $P \in A(F)$ such that $\operatorname{End}_{F}(A) P$ is a free $\operatorname{End}_{F}(A)$-module, the assumption that $r_{v}(P) \in r_{v}(\Lambda)$ for almost all $v \in \mathcal{O}_{F}$ implies that $a P \in \Lambda$. Later W. Gajda and K. Górnisiewicz GG09] showed that one can take $a=1$.

In [B09] the problem of linear dependence was answered affirmatively under the assumption that $\operatorname{End}_{F}(A) P$ is a free $\operatorname{End}_{F}(A)$-module and additionally that $\Lambda$ is a free $\mathbb{Z}$-module which has a $\mathbb{Z}$-basis linearly independent over $\operatorname{End}_{F}(A)$.

A. Perucca [Pe10] generalized some results of [B09] and GG09] to the case of a product of an abelian variety and a torus.

P. Jossen [Jo13] obtained a positive answer to the linear dependence problem for geometrically simple abelian varieties. His result is exact, not up to torsion.

Notice that A. Schinzel [Sch75] also gave the following nice counterexample, which shows that in Theorem 2.1 it is not enough to take only prime ideals $\mathfrak{p}$, except for $n=1$.

EXAMPLE 4.4. Consider the following exponential congruences:

$$
2^{x} 3^{y} \equiv 1 \bmod p, \quad 2^{y} 3^{z} \equiv 4 \bmod p .
$$

For $p=2$ we have an obvious solution $(x, y, z)=(0,1,0)$. For $p=3$ the solution is $(0,0,0)$. Let $p>3$. Choose a generator $\xi \bmod p$ of the cyclic group $\mathbb{F}_{p}^{\times}=(\mathbb{Z} / p)^{\times}$. For each $k \in \mathbb{F}_{p}^{\times}$let ind $k$ be such that $\xi^{\text {ind } k}=k$. Then the system 4.1 is equivalent to the following:

$$
\begin{aligned}
& x \text { ind } 2+y \text { ind } 3 \equiv 0 \bmod (p-1), \\
& y \text { ind } 2+z \text { ind } 3 \equiv 2 \text { ind } 2 \bmod (p-1) .
\end{aligned}
$$

Let $(t, z)$ be a solution of the auxiliary linear congruence

$$
t \frac{(\text { ind } 2)^{2}}{(\text { ind } 2, \text { ind } 3)}+z \text { ind } 3=2 \text { ind } 3 .
$$

Notice that since $\left(\frac{(\text { ind } 2)^{2}}{(\text { ind } 2 \text {,ind } 3)}\right.$, ind 3$) \mid 2$ ind 3 for any $p$, the congruence $\sqrt{5}$ is soluble for any $p$. Then $\left(x=\frac{-t \text { ind } 3}{(\operatorname{ind} 2, \text { ind } 3)}, y=\frac{t \text { ind } 2}{(\text { ind } 2, \text { ind } 3)}, z\right)$ is a solution of (4). On the other hand, there do not exist integers $x, y, z$ such that $2^{x} 3^{y}=1$ and $2^{y} 3^{z}=4$.

G. Banaszak and the second author [BK11] proved the following sufficient condition for the local-to-global principle for abelian varieties to hold modulo torsion. 
TheOREM 4.5. Let $F^{\prime} / F$ be a finite extension such that $A$ is isogenous over $F^{\prime}$ to

$$
A^{e_{1}} \times \cdots \times A^{e_{t}}
$$

with $A_{i} / F^{\prime}$ simple, pairwise nonisogenous abelian varieties. Assume that

$$
\operatorname{dim}_{\operatorname{End}_{F^{\prime}}\left(A_{i}\right)^{0}} H_{1}\left(A_{i}(\mathbb{C}) ; \mathbb{Q}\right) \geq e_{i}
$$

for each $1 \leq i \leq t$, where $\operatorname{End}_{F^{\prime}}\left(A_{i}\right)^{0}:=\operatorname{End}_{F^{\prime}}\left(A_{i}\right) \otimes \mathbb{Q}$. Fix $P \in A(F)$. Let $\Lambda$ be a subgroup of $A(F)$. If $r_{v}(P) \in r_{v}(\Lambda)$ for almost all primes $v$ of $\mathcal{O}_{F}$, then $P \in \Lambda+A(F)_{\text {tor. }}$ Moreover, if $A(F)_{\text {tor }} \subset \Lambda$, then the following conditions are equivalent:

(1) $P \in \Lambda$;

(2) $r_{v}(P) \in r_{v}(\Lambda)$ for almost all primes $v$ of $\mathcal{O}_{F}$.

The methods used in the proof of Theorem 4.3 are rather involved, but they also work for tori. Let $T / F$ be an algebraic torus. Let $F^{\prime} / F$ be a finite extension that splits $T$, i.e. $T \otimes_{F} F^{\prime} \simeq \mathbb{G}_{\mathrm{m}}^{e}$. Since $\operatorname{End}_{K}\left(\mathbb{G}_{\mathrm{m}}\right)=\mathbb{Z}$, for any $F^{\prime} \subset K \subset \bar{F}$ we see that the sufficient condition of Theorem 4.3 is $e \leq 1$. Hence Schinzel's counterexample is just off this condition. Using the methods of [BGK05] one can also get rid of the torsion ambiguity in this case.

In BK11, using elliptic curves with CM by $\mathbb{Z}[i]$, defined over $\mathbb{Q}$, of the form $E_{d}: y^{2}=x^{3}-d^{2} x$, it was shown that the linear dependence problem has a negative solution already for the surface $E_{d} \times E_{d}$, if $E_{d}(\mathbb{Q})$ has rank at least 2. But from the work of K. Rubin and A. Silverberg [RS02] we know that this rank can reach 6 .

Another nice example using elliptic curves without CM was given in [JP10.

Neither of these examples satisfies the assumptions of Theorem 4.3 .

We would like to pose the following problem

Problem 4.6. Remove the torsion ambiguity from Theorem 4.5.

5. Extension to $\mathrm{SL}(n)$. An extension of Theorem 3.1, from the context of the multiplicative group $\mathcal{O}^{\times}$to that of $\operatorname{SL}(2, \mathbb{Z})$, was given by $\mathrm{C}$. Khare and D. Prasad [KP04, Theorem 4]. Here is a simple extension to a general $n$ and inner automorphisms.

TheOREM 5.1. Let $\Gamma$ be a subgroup of $\operatorname{SL}(n, \mathcal{O})$ of finite index. Let $\phi: \Gamma \rightarrow \Gamma$ be a nontrivial homomorphism. Suppose there is an infinite set $S$ of prime ideals $\mathfrak{p}$ of $\mathcal{O}$ with the following property. For all $\mathfrak{p}$ in $S$, the homomorphism $\phi$ factors to give a homomorphism $\phi_{\mathfrak{p}}: \mathrm{SL}(n, \mathcal{O} / \mathfrak{p}) \rightarrow \mathrm{SL}(n, \mathcal{O} / \mathfrak{p})$, that is, the following diagram exists and is commutative: 


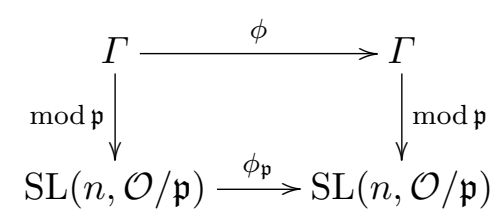

Moreover, suppose $\phi_{\mathfrak{p}}$ is inner, that is, $\phi_{\mathfrak{p}}(g)=\operatorname{Int}(x) g:=x g x^{-1}$ for some $x=x\left(\phi_{\mathfrak{p}}\right)$ in $\mathrm{GL}(n, \mathcal{O} / \mathfrak{p})$, for all $\mathfrak{p} \in S$. Then $\phi$ is an automorphism of $\Gamma$ which is the restriction to $\Gamma$ of the inner conjugation by an element of $\mathrm{GL}(n, F)$.

Proof. Set $B=\prod_{\mathfrak{p} \in S} \mathcal{O} / \mathfrak{p}$. The ring $\mathcal{O}$ embeds in $B$. Hence there is an injection $\mathrm{SL}(n, \mathcal{O}) \hookrightarrow \mathrm{SL}(n, B)$. So $\phi$ lies in a commutative diagram

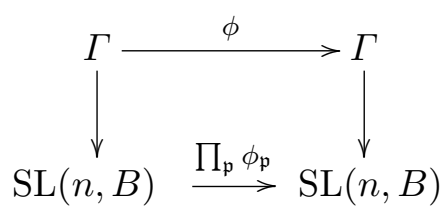

and it is locally inner. Hence the representation $\phi: \Gamma \rightarrow \mathrm{GL}(n, F)$ and the identity (natural embedding) representation id $: \Gamma \rightarrow \mathrm{GL}(n, F)$ have equal traces. Now it is well-known (see the lemma below) that two representations of a group $\Gamma$ in $\operatorname{GL}(n, \bar{F})$, with $\bar{F}$ an algebraically closed field of characteristic zero, one of which is irreducible, with equal traces, are equivalent, namely conjugate by some $x \in \operatorname{GL}(n, \bar{F})$. But id is irreducible, hence so is $\phi$, and $\phi$ and id are conjugate by an element of $\operatorname{GL}(n, \bar{F})$ : there is an $x \in \operatorname{GL}(n, \bar{F})$ such that $\phi(\gamma)=x \gamma x^{-1}$ for all $\gamma \in \Gamma$.

We claim $x$ can be taken in $\operatorname{GL}(n, F)$. As $\phi(\Gamma) \subset \operatorname{GL}(n, \mathcal{O})$, for each $\sigma \in \operatorname{Gal}(\bar{F} / F)$ we have $\phi(\gamma)=\sigma(x) \gamma \sigma(x)^{-1}$ for all $\gamma \in \Gamma$. Then $x \gamma x^{-1}=$ $\sigma(x) \gamma \sigma(x)^{-1}$, so $x_{\sigma}=x^{-1} \sigma(x)$ commutes with each element of $\Gamma$, a group of finite index in $\operatorname{GL}(n, \mathcal{O})$. Hence $x_{\sigma}$ lies in $Z(\bar{F})$, where $Z$ is the center of $\operatorname{GL}(n)$. So $\sigma \mapsto x_{\sigma}$ defines a cocycle in $\operatorname{ker}\left[H^{1}(F, Z) \rightarrow H^{1}(F, \operatorname{GL}(n))\right]$. But $H^{1}(F, Z)$ is trivial, by Hilbert's Theorem 90 , as $Z=\mathbb{G}_{\mathrm{m}}$ is the multiplicative group, as is $H^{1}(F, \mathrm{GL}(n))$. Hence there is a $z \in Z(\bar{F})$ with $x_{\sigma}=z \sigma(z)^{-1}$. So $x z$ lies in $\operatorname{GL}(n, F)$, and $\phi(\gamma)=x z \gamma(x z)^{-1}$ for all $\gamma \in \Gamma$. Hence $\phi(\Gamma)$ is conjugate to $\Gamma$ under $\operatorname{GL}(n, F)$. Consequently, $\phi$ is the restriction to $\Gamma$ of $\operatorname{Int}(x)$ for some $x \in \mathrm{GL}(n, F)$, and $\operatorname{Int}(x)$ takes $\Gamma$ to itself.

Above we have used the following, where the group $\Gamma$ is replaced by its group algebra $A=\bar{F}[\Gamma]$.

Lemma 5.2. Let $F$ be an algebraically closed field. Let $A$ be an algebra over $F$. Let $M$ and $N$ be A-modules of the same finite dimension over $F$. Suppose that they have the same character (trace of the elements) and that one of them is irreducible. Then they are isomorphic. 
Proof. Note first that we may assume that $A$ has finite dimension over $F$, by replacing it by its image in $\operatorname{End}(M) \times \operatorname{End}(N)$. Suppose $N$ is irreducible. Let $M^{\prime}$ be the semisimplification of $M$ (the direct sum of its simple JordanHölder subquotients). The character of $M^{\prime}$ is equal to that of $M$. By linear independence of characters (assume that $A$ is finite-dimensional, and has trivial radical; then it is a product of matrix algebras, the representations are obvious, and so is the linear independence of the characters), $M^{\prime}$ is isomorphic to the sum of $N$ and of other characters with multiplicities multiples of the characteristic of $F$. Because $N$ and $M^{\prime}$ have the same dimension, these multiplicities are all 0 . Hence $M^{\prime}$ is isomorphic to $N$. Hence $M$ is irreducible, and isomorphic to $N$.

REMARK 5.3. An automorphism of $\mathrm{SL}(n, \mathcal{O} / \mathfrak{p})$ is the composition of an inner automorphism from $\operatorname{GL}(n, \mathcal{O} / \mathfrak{p})$, the outer automorphism $g \mapsto{ }^{t} g^{-1}$, and Galois action. In the special case of $F=\mathbb{Q}$, the Galois action is trivial. In the special case of $n=2,{ }^{t} g^{-1}$ is conjugate to $g$ by $\left(\begin{array}{cc}0 & -1 \\ 1 & 0\end{array}\right)$. Hence in the case of $\Gamma \subset \mathrm{SL}(2, \mathbb{Z})$ the assumption that $\phi_{p}$ is inner is automatically satisfied. So in fact our local assumption is no different than the local assumption in [KP04, Theorem 4].

Note that since the only proper normal subgroup of $\mathrm{SL}(2, \mathbb{Z} / p)$ is the center $\{ \pm I\}$ when $p>3$, any nontrivial homomorphism of $\mathrm{SL}(2, \mathbb{Z} / p)$ to itself is onto if $p>3$ : the group $\operatorname{PSL}(2, \mathbb{Z} / p)$ has order half that of $\operatorname{SL}(2, \mathbb{Z} / p)$ when $p>2$, so it is not a subgroup of $\operatorname{SL}(2, \mathbb{Z} / p)$ when $p>3$, hence any surjection is then inner. When $p=3, \mathrm{SL}(2,3)=2 A_{4}=Q_{8}: C_{3}$, namely the quaternion group $Q_{8}$ of 8 elements is normal, with complement the cyclic 3-Sylow group $\left\langle\left(\begin{array}{ll}1 & 1 \\ 0 & 1\end{array}\right)\right\rangle$. When $p=2, \operatorname{SL}(2,2)=S_{3}$ has the normal subgroup $A_{3}$.

The case of $\mathrm{SL}(2, \mathbb{Z})$ is proven in $\mathrm{KP} 04$. They attribute their proof (which we extend to $\mathrm{SL}(n, \mathcal{O})$ ) to J.-P. Serre. We thank J.-P. Serre for the proof of Lemma 5.2, and critique.

Acknowledgements. Y. F. was partially supported by the Simons Foundation grant \#317731. He thanks Szczecin University for hospitality when most of this work was done.

P. K. was partially supported by an NCN grant.

\section{References}

[B09] G. Banaszak, On a Hasse principle in Mordell-Weil groups, C. R. Math. Acad. Sci. Paris 347 (2009), 709-714.

[BK11] G. Banaszak and P. Krason, On arithmetic in Mordell-Weil groups, Acta Arith. 150 (2011), 315-337. 
[BGK03] G. Banaszak, W. Gajda and P. Krason, A support problem for the intermediate Jacobians of $\ell$-adic representations, J. Number Theory 100 (2003), 133-168.

[BGK05] G. Banaszak, W. Gajda and P. Krasoń, Detecting linear dependence by reduction maps, J. Number Theory 115 (2005), 322-342.

[Bi67] B. Birch, Cyclotomic fields and Kummer extensions, in: J. W. S. Cassels and A. Fröhlich (eds.), Algebraic Number Theory, Academic Press, 1967, 85-93.

[BLR90] S. Bosch, W. Lütkebomert and M. Raynaud, Néron Models, Ergeb. Math. Grenzgeb. 21, Springer, 1990.

[CRS97] C. Corrales-Rodrigáñez and R. Schoof, The support problem and its elliptic analogue, J. Number Theory 64 (1997), 276-290.

[GG09] W. Gajda and K. Górnisiewicz, Linear dependence in Mordell-Weil groups, J. Reine Angew. Math. 630 (2009), 219-233.

[Jo13] P. Jossen, Detecting linear dependence on an abelian variety via reduction maps, Comment. Math. Helv. 88 (2013), 323-352.

[JP10] P. Jossen and A. Perruca, A counterexample to the local-global principle of linear dependence for abelian varieties, C. R. Math. Acad. Sci. Paris 348 (2010), 9-10.

[Kh03] C. Khare, Compatible systems of mod $p$ Galois representations and Hecke characters, Math. Res. Lett. 10 (2003), 71-83.

[KP04] C. Khare and D. Prasad, Reduction of homomorphisms mod $p$ and algebraicity, J. Number Theory 105 (2004), 322-332.

[Ko03] E. Kowalski, Some local-global applications of Kummer theory, Manuscripta Math. 111 (2003), 105-139.

[La03] M. Larsen, The support problem for abelian varieties, J. Number Theory 101 (2003), 398-403.

[Ne99] J. Neukirch, Algebraic Number Theory, Grundlehren Math. Wiss. 322, Springer, 1999.

[Pe10] A. Perucca, On the problem of detecting linear dependence for products of abelian varieties and tori, Acta Arith. 142 (2010), 119-128.

[Rot09] J. Rotman, Introduction to Homological Algebra, Universitext, Springer, 2009.

[RS02] K. Rubin and A. Silverberg, Ranks of elliptic curves, Bull. Amer. Math. Soc. 39 (2002), 455-474.

[Sch75] A. Schinzel, On power residues and exponential congruences, Acta Arith. 27 (1975), 397-420.

[Se97] J.-P. Serre, Galois Cohomology, Springer, 1997.

[Sk37] Th. Skolem, Anwendung exponentieller Kongruenzen zum Beweis der Unlösbarkeit gewisser diophantischer Gleichungen, Avh. Norske Vid.-Akad. Oslo 1937, no. $12,16 \mathrm{pp}$.

[We03] T. Weston, Kummer theory of abelian varieties and reductions of Mordell-Weil groups, Acta Arith. 110 (2003), 77-88.

Yuval Z. Flicker

Ariel University

Ariel 40700, Israel

and

The Ohio State University

Columbus, OH 43210, U.S.A.

E-mail: yzflicker@gmail.com
Piotr Krasoń

University of Szczecin

Wielkopolska 15

70-451 Szczecin, Poland

E-mail: piotrkras26@gmail.com 